\title{
Cryogenic Lorentz TEM study of a Berezinskii-Kosterlitz-Thouless phase transition in the quasi-two-dimensional ferromagnet $\mathrm{K}_{2} \mathrm{CuF}_{4}$ ?
}

Yoshihiko Togawa ${ }^{1}$, Tetsuya Akashi $^{2}$, Hiroto Kasai ${ }^{3}$, Gary Paterson ${ }^{4}$, Stephen McVitie ${ }^{4}$, Yusuke Kousaka $^{1}$, Hiroyuki Shinada ${ }^{2}$, Jun-ichiro Kishine ${ }^{5}$ and Jun Akimitsu ${ }^{6}$

${ }^{1}$ Osaka Prefecture University, Sakai, Osaka, Japan, ${ }^{2}$ Research \& Development Group, Hitachi, Ltd., Hatoyama, Saitama, Japan, ${ }^{3}$ Research and Development Group, Hitachi Ltd., Hatoyama, Saitama, Japan, ${ }^{4}$ University of Glasgow, Glasgow, Scotland, United Kingdom, ${ }^{5}$ The Open University of Japan, Chiba, Japan, ${ }^{6}$ Okayama University, Okayama, Japan

The dimensionality of a system has a strong influence on its structure and dynamics. Indeed, the nature of the phase transition in a low-dimensional system has attracted considerable interest for many decades. One of the celebrated works in the field is the proposal of the topological ground state in the twodimensional (2D) spin system, which was made by Kosterlitz and Thouless [1] and awarded the Nobel Prize in Physics 2016.

It is well established that no ordered phase and no spontaneous magnetization appear at a finite temperature in one and two dimensions of Heisenberg and $X Y$ spins, while a divergence of susceptibility may occur at a finite temperature as an indication of the phase transition in the 2D system of Heisenberg spins. This puzzling situation was resolved by Kosterlitz and Thouless. They showed that the phase transition occurs at a finite temperature given by $T_{\mathrm{KT}}$ where quasi long-range order emerges in the 2D system of $X Y$ spins through the formation and unbinding of vortex-antivortex pairs. Berezinskii independently pointed out the importance of vortex excitations in the $X Y$ model. The 2D $X Y$ model enables magnetic vortex and antivortex excitations and undergoes a phase transition of vortex and antivortex unbinding, referred to as BKT phase transition.

The existence of the BKT phase transition was experimentally demonstrated in a thin 2D film of Helium as well as in a superconducting Josephson network. However, to our surprise, no reliable experimental demonstration of the BKT phase transition has been performed in a magnetic system. This is mainly due to the following reasons: 1) 2D $X Y$ magnets appropriate for the BKT phase transition were not readily available. 2) The techniques commonly used in the study of these materials were not capable of directly observing the BKT phenomena.

In this study, using cryogenic Lorentz transmission electron microscopy, we investigate the temperature evolution of magnetic structures in single crystal samples of $\mathrm{K}_{2} \mathrm{CuF} 4$ with a magnetic phase transition at $6.1 \mathrm{~K}$ [2]. This material is one of the candidates for a 2D $X Y$ magnet that may exhibit the BKT phase transition.

A fine magnetic stripe pattern was found with a period of about $120 \mathrm{~nm}$ in a direction along the $c$-axis below $7.3 \mathrm{~K}$ in a thin sample with the $c$-axis in the plane. Magnetic columns of vortices and antivortices with a separation of about three micrometers were observed below $6 \mathrm{~K}$ in a $c$-plane sample approximately $150 \mathrm{~nm}$ in thickness. The formation of two different types of magnetic structures at different threshold temperatures is likely to be consistent with a picture of two-step dimensional crossover in spin and real spaces previously derived from magnetization and neutron experiments in $\mathrm{K}_{2} \mathrm{CuF} 4$. 
These results indicate how the $2 \mathrm{D} X Y$ character of $\mathrm{K}_{2} \mathrm{CuF}_{4}$ is incorporated in three-dimensional magnetic structures. Based on the experimental observations, we discuss the lengthscale of film thickness appropriate for expanding the 2D $X Y$ regime and generating the robust BKT excitations. We expect our study to be an important step in realizing the BKT phase transition in a real magnetic system.

We acknowledge support from Grants-in-Aid for Scientific Research (Nos. 17H02767, 17H02923). This work includes the results obtained by using the research equipment shared in the MEXT Project for promoting public utilization of advanced research infrastructure (Program for supporting introduction of the new sharing system: Grant Number JPMXS0410500020) and the United Kingdom research council EPSRC (grant number EP/M024423/1).

\section{References}

[1] J. M. Kosterlitz and D. J. Thouless, J. Phys. C6,1181 (1973).

[2] Y. Togawa et al., J. Phys. Soc. Jpn., 90, 014702 (2021). 